\title{
Evaluation of 3D printed microfluidic networks to study fluid flow in rocks
}

\author{
Seyed Mahdi Mousavi ${ }^{1}$, Saeid Sadeghnejad ${ }^{1,{ }^{*}}$, and Mehdi Ostadhassan ${ }^{2,3}$ \\ ${ }^{1}$ Department of Petroleum Engineering, Faculty of Chemical Engineering, Tarbiat Modares University, Tehran, Iran \\ ${ }^{2}$ Key Laboratory of Continental Shale Hydrocarbon Accumulation and Efficient Development, Ministry of Education, Northeast \\ Petroleum University, 163318 Daqing, China \\ ${ }^{3}$ Department of Petroleum Engineering, Amirkabir University of Technology, Tehran, Iran
}

Received: 18 December 2020 / Accepted: 12 May 2021

\begin{abstract}
Visualizing fluid flow in porous media can provide a better understanding of transport phenomena at the pore scale. In this regard, transparent micromodels are suitable tools to investigate fluid flow in porous media. However, using glass as the primary material makes them inappropriate for predicting the natural behavior of rocks. Moreover, constructing these micromodels is time-consuming via conventional methods. Thus, an alternative approach can be to employ 3D printing technology to fabricate representative porous media. This study investigates fluid flow processes through a transparent microfluidic device based on a complex porous geometry (natural rock) using digital-light processing printing technology. Unlike previous studies, this one has focused on manufacturing repeatability. This micromodel, like a custom-built transparent cell, is capable of modeling single and multiphase transport phenomena. First, the tomographic data of a carbonate rock sample is segmented and 3D printed by a digital-light processing printer. Two miscible and immiscible tracer injection experiments are performed on the printed microfluidic media, while the experiments are verified with the same boundary conditions using a CFD simulator. The comparison of the results is based on Structural Similarity Index Measure (SSIM), where in both miscible and immiscible experiments, more than $80 \%$ SSIM is achieved. This confirms the reliability of printing methodology for manufacturing reusable microfluidic models as a promising and reliable tool for visual investigation of fluid flow in porous media. Ultimately, this study presents a novel comprehensive framework for manufacturing 2.5D realistic microfluidic devices (micromodels) from pore-scale rock images that are validated through CFD simulations.
\end{abstract}

\section{Introduction}

Microfluidic systems are usually used in multidisciplinary studies to simulate transport processes and reveal relevant underlying mechanisms in details in porous media, and petroleum industry is not an exception [1-3]. These microfluidic networks, also known as fluidic micromodels, are employed to visualize fluid flow through porous-like networks. Manufacturing microfluidic-channels are mostly based on subtractive and additive fabrication methods [4].

Micromodels have been manufactured directly using glass beads in the Hele-Shaw flow approach [5] or by etching pore morphology on glasses/silicon wafers [6-8]. For example, Meisenheimer et al. [9] used a simplified porous medium of soda-lime glass beads to investigate two-phase flow. To etch the flow pattern on a siliceous glass, either laser-etching technique or wet etching approach can be implemented where laser beam (e.g., a $\mathrm{CO}_{2}$ laser device

\footnotetext{
* Corresponding author: sadeghnejad@modares.ac.ir
}

[10]) and acid is used respectively on silicon or silicon plates to develop microfluidic patterns [11]. In both approaches, a second glass plate is placed on top of the etched glass to create an enclosed pore space. Finally, heat treatment for thermal fusion process in an oven would be necessary to ensure the plates are fully attached and sealed [12]. Sharifipour et al. [13] studied the effect of clay swelling on the oil recovery factor in a porous medium using micromodels, where the device was made through this process. Furthermore, a laser-etched rock on-chip was also used to resemble the pore space and fracture geometries [14]. Martel et al. [15] utilized the physical properties of fractured bedrocks to design a micromodel etched by hydrofluoric acid on two glass plates. This setup enabled them to understand the critical parameters for an efficient recovery of nonaqueous phase liquids using surfactant solutions. Although the popularity, the conventional glass micromodel manufacturing is a time-consuming and tedious process [16]. Moreover, since non-geological materials are used to build them (e.g., glass, plastic), their properties 
cannot represent most rock samples. In contrast, rock petrophysical properties play an integral role in fluid flow. Among these properties, wettability is one that should be adjusted in such models (e.g., by micromodel aging), enforcing additional steps in preparing the models [17].

To address the above issues, researchers have used natural rocks to fabricate microfluidic pore-network models [18-20]. In a study, a 500- $\mu \mathrm{m}$ rock piece, mounted between two polydimethylsiloxane plates, was used as a microfluidic device [21]. Recently, Teimouri et al. [22] designed and manufactured a transparent cell consisting of two plexiglass plates around a carbonate rock sample to investigate the conformance control during $\mathrm{pH}$-sensitive polymer flooding in a fractured media. The transparent sealing rubber enabled them to photograph the rock surface easily during the experiments. It should be noted that preparing microfluidic models from natural rock samples is challenging since the specimens should be thick enough to avoid chipping during the fabrication, but thin enough to precisely visualize the fluid flow over the entire grooved depth [23, 24]. The main disadvantage of such setups is limitations in their reusability which contradicts the reason for employing such models. This means they can be used solely once for a single run during destructive experiments (e.g., reactive flow), which makes repeating the same experiments impossible.

The micromodel mineral coating approach is receiving increased attention due to providing compositional similarity with natural rocks. For example, Song and Kovscek [25] coated a microfluidic device with clay minerals to mimic a natural rock surface. This method ensured real-time observations of fluid-solid interactions in pore networks. They used this microfluidic model to analyze the effects of brine injection on clay detachments. In a separate study, micromodels were coated with high swelling clay minerals to model the behavior of sandstones that contain swelling clays [13]. Other researchers later used a layer-by-layer self-assembly technique to coat the surface of channels in microfluidic chips with geomaterials [26]. The main challenge of coated micromodels is to make sure a homogeneous layer of coating material with sufficient thickness on the micromodel surface is applied, though they cannot be used in destructive experiments (e.g., acid injection, formation damage).

All of the above fabrication methods try to ensure the resemblance of chemical and physical properties of the inner surfaces to the natural rocks, but they failed to ensure the reusability. Therefore, 3D printing is an alternative technology to build two- or three-dimensional objects from a computer-aided design model by adding materials in layers, which is known as additive manufacturing. Hull [27] developed commercial stereolithography technology for the first time. Additive manufacturing has been applied in different areas of science, including prototyping [28], automotive industry [29], jewelry [30], construction [31], aerospace [32], medicine [33], and petroleum industries [34, 35]. 3D printing is a relatively cheap, rapid, and easy process to analyze fluid flow in porous media $[10,16]$, which is often faster, cheaper, and less complicated than other microfluidic fabrication methods. Besides, 3D printing technology allows us to alter numerous factors, such as dimension, surface structure and roughness, wettability, and chemical properties of microfluidic domains [36, 37]. The unique feature of this fabrication method is the physical model reproducibility, where several identical copies of the same porous medium can be created which is necessary for destructive experiments.

In several studies, 3D printed core plugs were fabricated to assess the behavior of porous media [38-40]. For example, [41] used gypsum-powder-based 3D printed synthetic samples to study the pore structures (e.g., porosity, pore size distribution, anisotropy) of core plugs. In another study, two 3D printed cylindrical samples with different sizes were fabricated by gypsum powder to investigate the feasibility and reliability of artificial specimens in terms of rock geo-mechanical behavior [42]. Moreover, Kong et al. [43] evaluated the matrix pore structure and alterations after uniaxial compressive strength tests. They examined the overall performance of 3D printing technology in terms of its limitations and flaws. Besides, they tried to study particle and pore structure characterization of 3D printed silica samples using 2D and 3D imaging techniques [44]. A new approach for $3 \mathrm{D}$ reconstruction of a rock sample from Computed Tomography (CT) data of a Berea sandstone core plug was proposed, and the resulting 3D image was utilized for printing samples with various printing technologies for comparison purposes [45]. Moreover, the same researchers explained a workflow for creating accurate $3 \mathrm{D}$ printed core samples using a machine-learning image processing approach based on the CT data [46]. The porescale waterflooding experiments were also carried out on mixed-wetted natural sandstone along with a $3 \mathrm{D}$ printed prototype using $\mu \mathrm{CT}$ data [47]. The authors investigated wettability and pore geometry effects on the morphology of the residual oil block. Hasiuk [48] characterized the pore networks using a 3D printed solid porous proxies fabricated by a powder-based 3D printer. Printed rock analogs were manufactured with steel, aluminum, and gypsum powders. The shortcoming of these models is that the saturation distribution as well as fluid pattern through these 3D printed models cannot be investigated visually regardless of the method, (e.g., microscopy) and other advanced techniques (e.g., CT scanning or nuclear magnetic resonance) should be implemented. Thus, there is still a need for microfluidic devices that can be examined visually, which is necessary to better understand the processes that take place during EOR.

Furthermore, 3D printing technology to fabricate simple-shaped micromodels has also been utilized as well, where Watson et al. [16] created a 2D printed micromodel to simulate single-phase flow. Yang et al. [49] fabricated two transparent geometries to study the multiphase flow in a fracture-vug medium using 3D printing technology. They monitored the flow process using the backlight visualization method. The capillary flow and transport phenomena in micron-sized porous media made out of polymer were also investigated using powder-bed fusion printing technology [50]. Ahkami et al. [51] conducted $\mathrm{CO}_{2}$ flooding experiments on a multiJet 3D printed fractured porous medium, where simple square pillars were implemented as 
fractures within the printed flow cell. One major drawback of these above studies was selecting a very simple pore shape geometry (e.g., lines as fractures) for the microfluidic device that might not be truly representative of a real pore geometry.

In this study, an improved manufacturing method to build microfluidic devices, including complex geometries is developed via 3D printing to investigate multiphase (i.e., miscible and immiscible) pore-scale flow. This complex printed model is derived from CT images of a carbonate rock sample as an input. By implementing image-processing techniques, water breakthrough time, fluid flow path, and the recovery factors are directly determined from the experiments. Moreover, numerical flow modeling is used to evaluate the accuracy of micromodel printing via Structural Similarity Index Measure (SSIM). Collectively, this study offers a novel methodology to analyze fluid flow in a porous medium by fabricating a visual repeatable and reusable microfluidic device. To the best of our knowledge, this is the first time that such an integrated guideline is being introduced for manufacturing 2.5D realistic microfluidic devices (micromodels) from pore-scale rock images where the quality of the printed model is verified by CFD simulations and results can be examined visually. Such a microfluidic device with controlled properties can be used to support our understanding of EOR processes. In other words, this simple model, which is created under controlled conditions, would be a good replacement for destructive laboratory tests and provides a visual tool for examining pore-scale phenomena during EOR processes (e.g., displacement and sweep mechanisms).

\section{Methods and materials}

\subsection{From computed tomography to proxy model}

Figure 1 depicts the general workflow in this study. First, CT scanning images $[52,53]$ were converted to binary series using an image processing procedure via ImageJ, an opensource platform [54] (Fig. 2a). A Region Of Interest (ROI) with a size of $400 \times 400 \times 400$ voxels was selected from printing and further analysis. A non-local mean filter was applied to the CT images to reduce the noise. Then, the $K$-means clustering-based thresholding algorithm [55] was used for image segmentation (Fig. 2b). The result of this step is a binary image, wherein pores are distinguished from the grains. In the next step, the isolated pores, as well as the pores on the edges of the image (to prevent the fluid leakage), were removed. The image resolution was decreased using the bilinear interpolation method to fit to the resolution of the 3D printer device. Next, an input and an output flow ports were designed in diagonal corners of the microfluidic setup (Fig. 2c). In the final step, the selected image is converted into a 3D model for printing as well as numerical simulations (Fig. 2d). The final dimensions of the printed model is $50 \times 50 \times 5 \mathrm{~mm}$ with a voxel size of $250 \times 250 \times 100 \mu \mathrm{m}$. It is worth mentioning that the resolution decreasing of the original micro-CT image to the 3D printer resolution definitely changes the properties of porous media. To avoid any inconsistency,

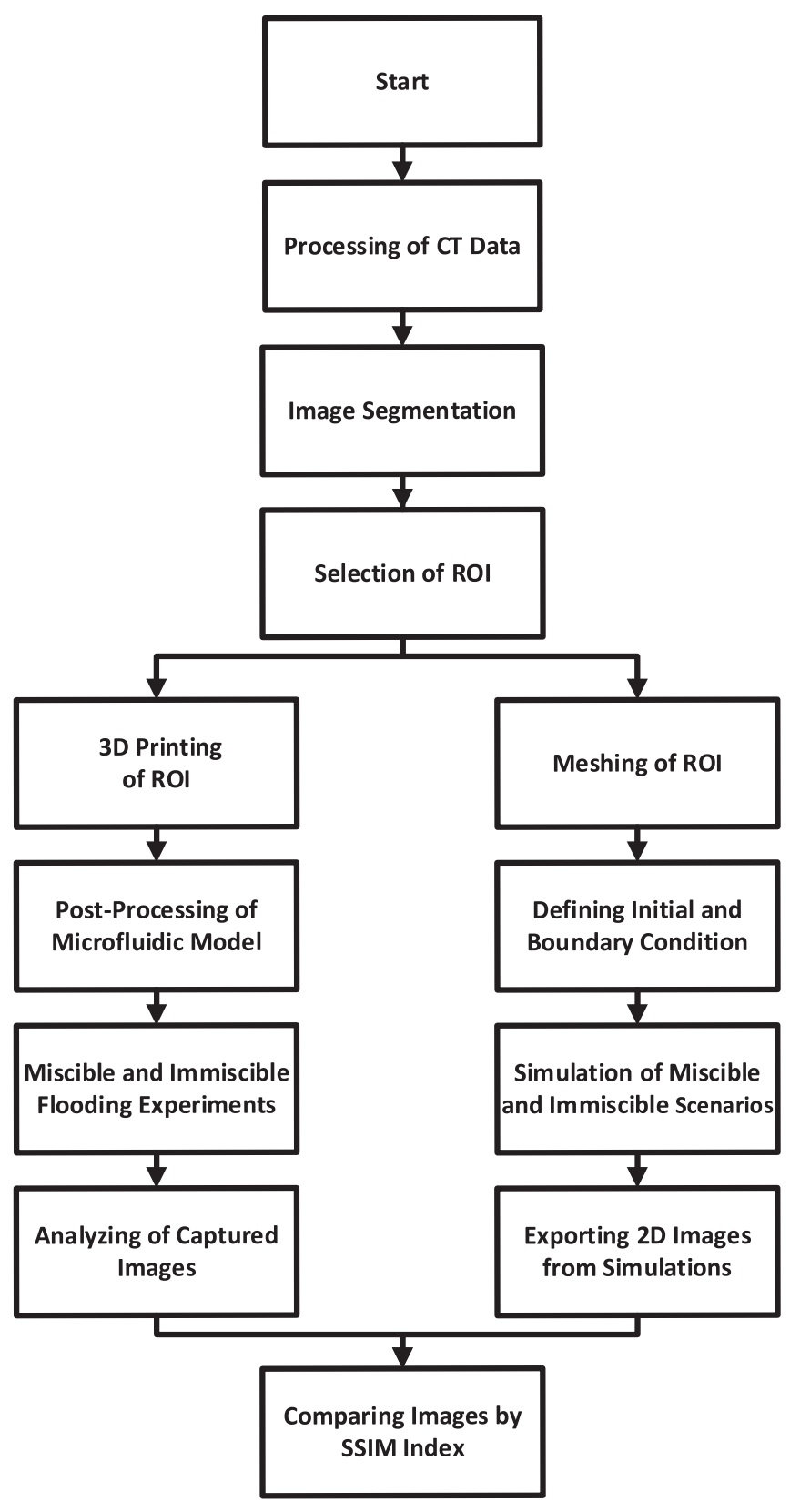

Fig. 1. The workflow of this study.

all further simulations, laboratory experiments, and their related comparisons are performed on the new lower resolution. It should be noted that by $3 \mathrm{D}$ printing technology advancement and using printers with higher resolutions, more sophisticated micromodels can be built until an analogue resembling to a natural rock sample with entirely controlled properties can be created. However, the generality of the introduced integrated guideline for manufacturing $2.5 \mathrm{D}$ microfluidic devices remains the same.

\subsection{Microfluidic model fabrication}

To print the microfluidic model, Digital Light Processing (DLP) that uses photopolymers for printing was utilized. 


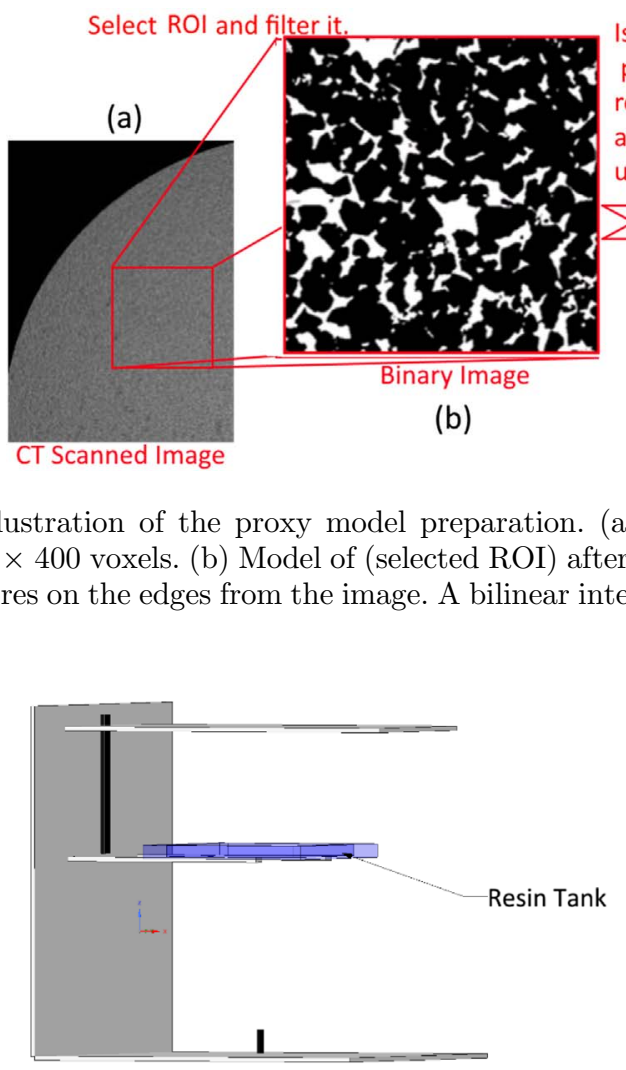

(a)

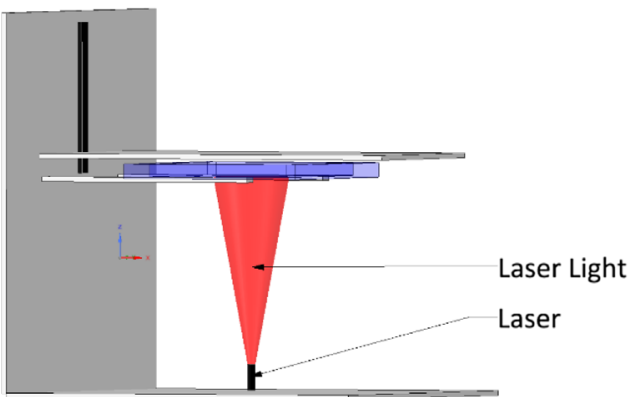

(c)

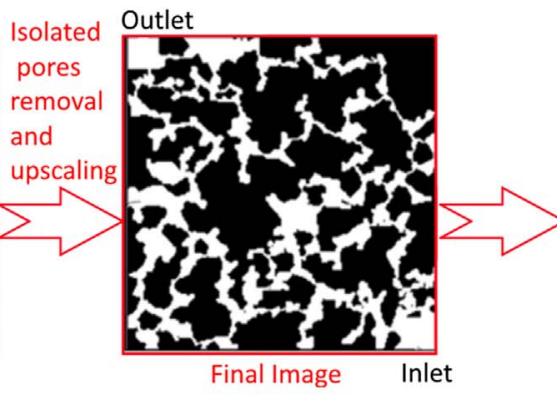

(c)

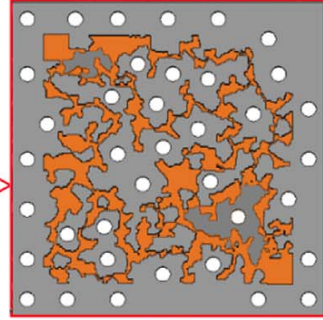

Created 3D model

(d)

Fig. 2. Illustration of the proxy model preparation. (a) CT scanning image. Select A Region Of Interest (ROI) with a size of $400 \times 400 \times 400$ voxels. (b) Model of (selected ROI) after the image segmentation. (c) Model of (b) after removing the isolated pores and the pores on the edges from the image. A bilinear interpolation method was also performed to increase connectivity. (d) Final 3D image.

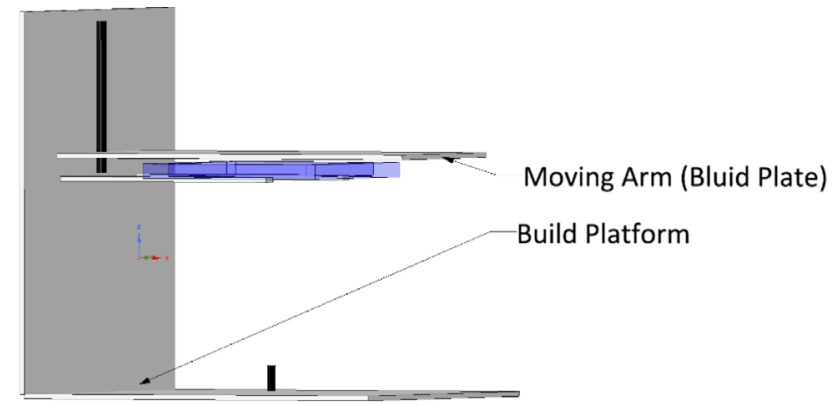

(b)

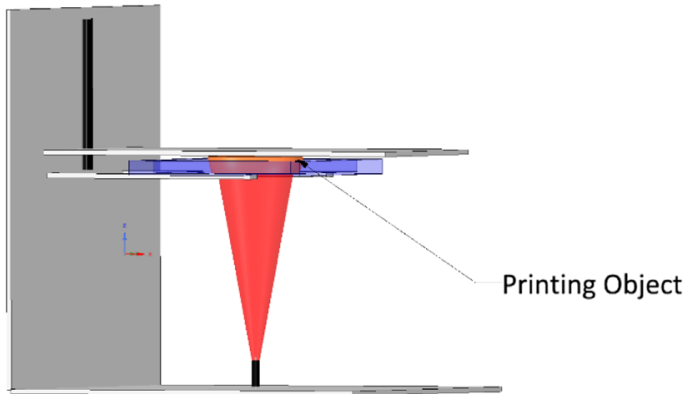

(d)

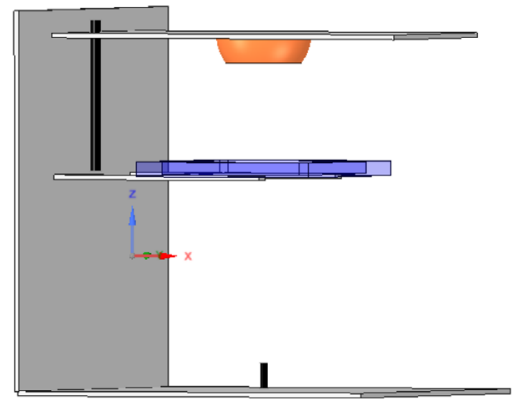

(e)

Fig. 3. 3D printing procedure. (a) Initial position. (b) The moving arm moves toward the heated resin tank and touches the resin tank bottom (only a small height is left between the build plate and the resin tank bottom). (c) Then the laser beam solidifies the liquid resin captured inside of this height. (d) After the layer consolidating, the moving arm moves upward. (e) The moving arm returns to the initial position, and this process is repeated subsequently to form up the printing object ultimately. 
These materials are initially in a liquid state and become harden by using a light source [30]. This printer can fabricate objects layer-by-layer and harden layers by laser beams on the surface of a photo-sensitive liquid resin layer (Fig. 3). The implemented laser (Fig. 3a) is generated by the digital light projector of the $3 \mathrm{D}$ printer. The liquid resin is inside of a tank (Fig. 3a) with a transparent bottom so that the projected laser can reach the resin and cure it (Fig. 3c). The build plate (Fig. 3b) simply sticks to the printed object's surface during printing and moves in the $z$-direction during the printing process. The build plate's moving arm descends into the resin and stops at a small height above the bottom of the tank (Fig. 3b). This height is specified by the thickness of the layer that should be printed. In the next stage, the digital light projector illuminates through the bottom of the resin tank (Fig. 3c) only on the model's particular pixels. This projection cures the resin in those areas into a solid. The cured resin creates the first layer of the proxy model (Fig. 3d), while the remaining uncured pixels are drained from the model. Then, the build platform moves upward to allow for another layer of resin to be cured (Fig. 3e). This process continues until the entire structure of the printing object is fabricated.

A Quantum Queen stereolithography 3D printer (Persia Company) was used to fabricate the proxy model of the rock sample. The resolution in the 3D model was $250 \mu \mathrm{m}$ in the $x$ and $y$ directions, and the layer thickness was adjusted to $100 \mu \mathrm{m}$. Figure 4 depicts the final printed microfluidic media. Table 1 summarizes the $3 \mathrm{D}$ printed microfluidic model and the materials specification in detail.

Post-processing plays an essential role in fabricating $3 \mathrm{D}$ printed models [38]. It consists of two steps, (1) UV curing and (2) removing the supports and ethanol flushing. UV curing makes the fresh 3D printed microfluidic model to solidify. Because of the Gaussian-shape of the UV light (the UV light intensity decreases at the corners) [56], if the model size is precisely equal to the platform size, the edges will not harden appropriately. Increasing the light intensity to higher values cannot resolve this problem, as the center of the model turns out to be fragile. Therefore, it is suggested that the size of the 3D CAD model be always smaller than the build platform. The model is printed vertically to reduce the adhesion between the printed microfluidic model and the build platform. It also prevents bending and crumbling during the printing process. In our model, several supports were printed with the model in a vertical direction, which must be removed (by the ethanol washing) after printing is accomplished (Fig. 5).

When curing is done, to seal the microfluidic model and avoid fluid escaping from the model during the experiments, two transparent plexiglass sheets (Fig. 6a) were attached to both faces of the printed microfluidic model using screws and bolts (Fig. 6b). It should be mentioned that the screw holes were located in the solid areas of the pore network (i.e., outside of pore space) (Fig. 6c) and did not interference with the pore space. To ensure the model is fully sealed, a PolyDiMethylSiloxane (PDMS) silicone gel was applied on the upper side (solid areas and not pores) of microfluidic device. The plexiglass sheet was then fixed on the upside of the printed model using screws and bolts.

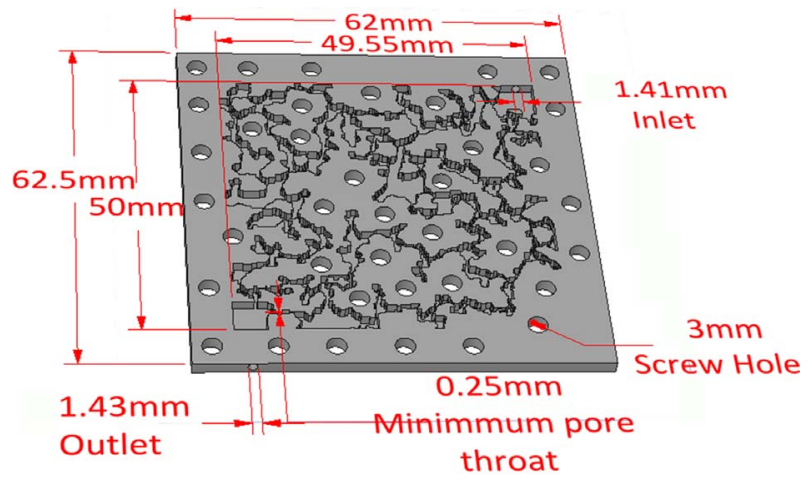

(a)

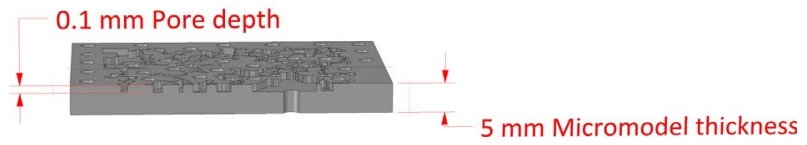

(b)

Fig. 4. The 3D CAD model of the microfluidic model of Figure 2d.

Table 1. The 3D printer and the materials used in this study.

\begin{tabular}{lc}
\hline Parameter & Value \\
\hline Build volume & $145 \times 145 \times 179 \mathrm{~mm}$ \\
Material & Wanhao transparent resin \\
Accuracy in $x$ - and $y$-directions & 250 microns \\
Layer thickness & 100 microns \\
Laser spot size & 140 microns \\
\hline
\end{tabular}

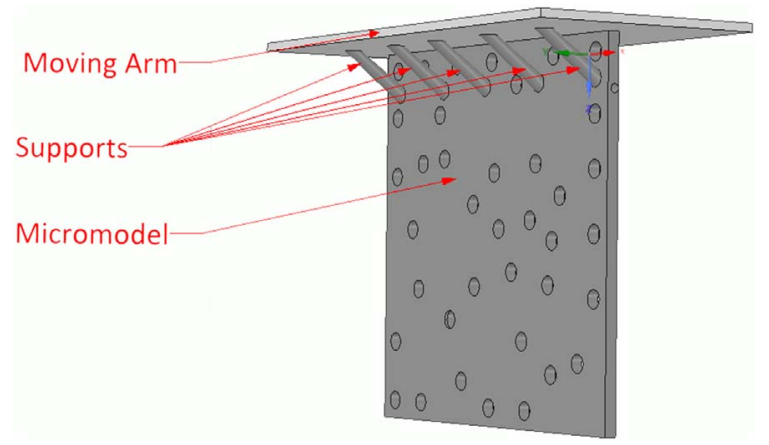

Fig. 5. 3D scheme of the microfluidic model and its supports on the build platform.

PDMS, also known as dimethylpolysiloxane or dimethicone, belongs to a group of polymeric organosilicon compounds that are commonly referred to as silicones [57]. This silicone gel layer prevents fluid scaping from the area between plexiglass and solid voxels of the printed model and is not in direct contact with pore areas. The transparency of the plexiglass sheets and the 3D printed block 


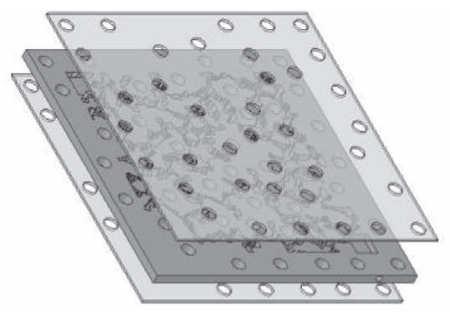

(a)

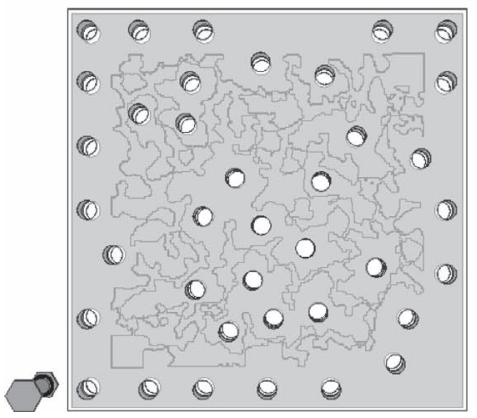

(c)

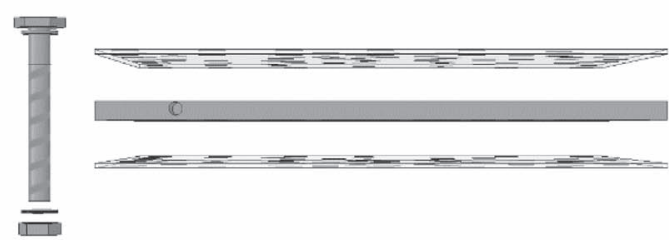

(b)

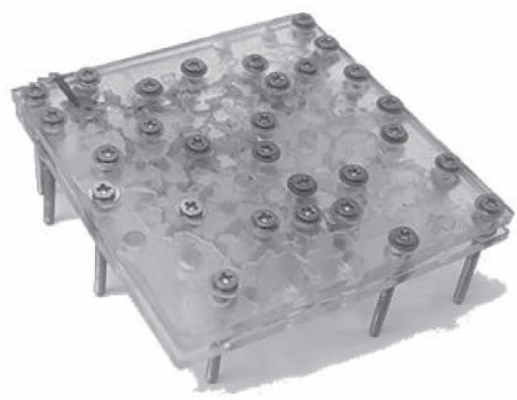

(d)

Fig. 6. The microfluidic model, (a) $3 \mathrm{D}$ scheme, (b) $2 \mathrm{D}$ scheme, side view, (c) $2 \mathrm{D}$ scheme, top view, (d) final microfluidic model.

Table 2. Properties of injected fluids.

\begin{tabular}{lccc}
\hline Material name & Company & Density at $20{ }^{\circ} \mathrm{C}\left(\mathrm{kg} / \mathrm{m}^{3}\right)$ & Viscosity at $20{ }^{\circ} \mathrm{C} \mathrm{Pas}$ \\
\hline n-heptane & MilliporeSigma & 680 & 0.0049 \\
Toluene & MilliporeSigma & 870 & 0.0043 \\
Tracer $\left(\mathrm{C}_{16} \mathrm{H}_{10} \mathrm{~N}_{2} \mathrm{O}_{2}\right)$ & Synthesized & 998 & 0.0009 \\
\hline
\end{tabular}

(i.e., microfluidic model) allows us to access the porous medium for photography purposes easily or cleaning it after each experiment is done (Fig. 6d).

\subsection{Protocol of experiments}

Two scenarios were considered in the experiments, miscible (i.e., tracer test) and immiscible (waterflooding into a saturated microfluidic model with synthetic oil) injection. The synthetic oil in this study is a heptane-toluene, $50-50 \%$ solution. The tracer was a very dilute weak Indigo dye $\left(\mathrm{C}_{16} \mathrm{H}_{10} \mathrm{~N}_{2} \mathrm{O}_{2}\right)$ solution. Indigo dye is used because of its natural organic nature and with a distinctive blue color [58]. Because of using a very dilute concentration of the dye, its effect on surface tension was ignored. Table 2 summarizes the properties of the fluids. The overall procedure for both laboratory experiments is as follows: (1) the microfluidic model is saturated with the primary fluid, (2) the displacing fluid is injected, (3) a digital camera dynamically captures the flow paths at different time steps, and (4) the captured images are processed for further analyses. Experiments are continued until the displacing fluid front breaks through the outlet port of the microfluidic model.
Figure 7 illustrates the schematic of the experimental setup. The microfluidic model is saturated with deionized water and synthesized oil in the miscible and immiscible injection scenarios, respectively. Then, the second fluid is injected through the inlet ports into the printed model with a constant flow rate of $2 \pm 0.01 \mathrm{~mL} / \mathrm{h}$. To distinguish between the displacing and the displaced fluids, blue dye as a tracer in our experiments were employed. Both miscible and immiscible injection scenarios were repeated twice to verify the results. The injection process is captured by a camera and converted to binary images through image processing techniques. To do so, first, a non-local mean filter is applied to all images to suppress the noise. The images are then converted to black and white (binary) segments by the $\mathrm{K}$-means Clustering-Based thresholding plugin in the ImageJ toolbox.

The contact angle between the synthetic oil and the printed microfluidic model and the plexiglass sheets were measured via the sessile drop technique (Fig. 8). The oil contact angle data for the plexiglass sheets and printed microfluidic models were measured $112^{\circ}$ and $122^{\circ}$, respectively. The contact angles of water fluid are $66^{\circ}$ and $55^{\circ}$ for the plexiglass sheet and printed microfluidic models, 


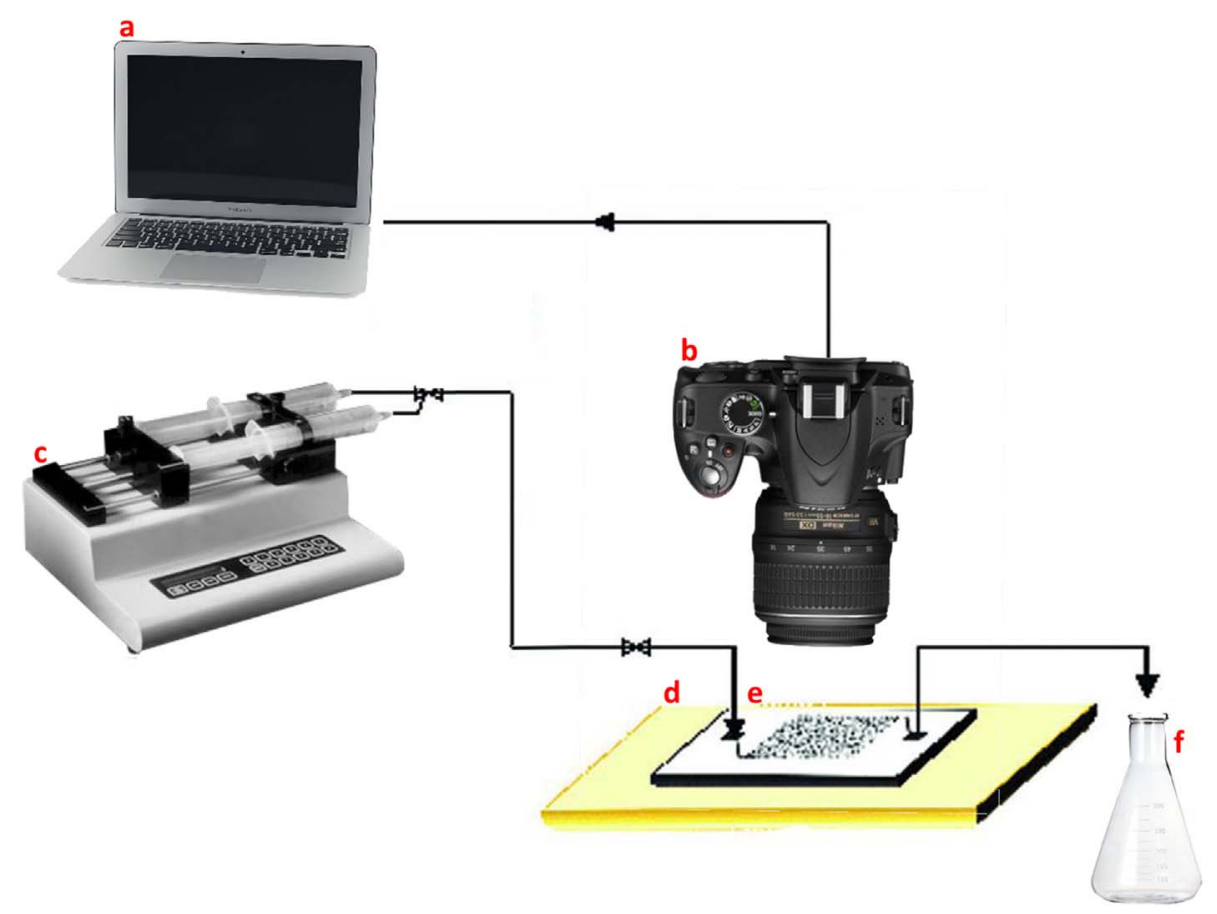

Fig. 7. Schematic diagram of the experimental setup. (a) Computer, (b) camera, (c) syringe pump: oil and tracer containers, (d) LED light source, (e) 3D printed microfluidic model, (f) sample collector.

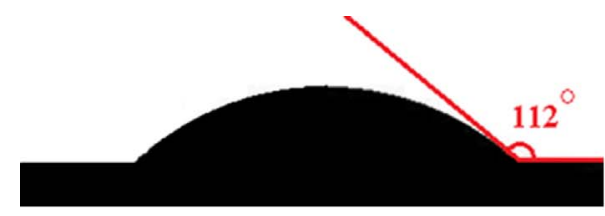

(a1)

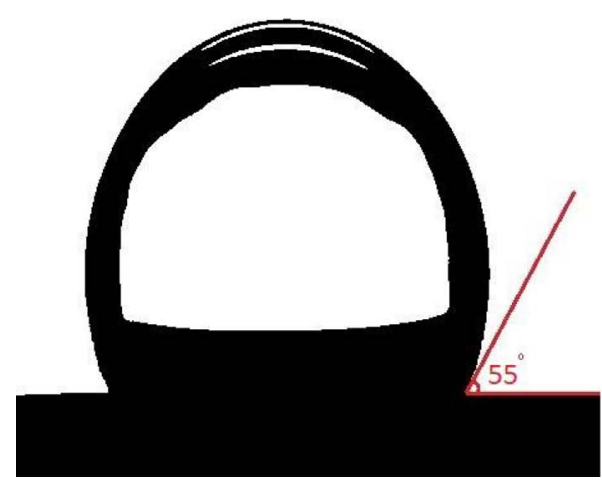

(b1)

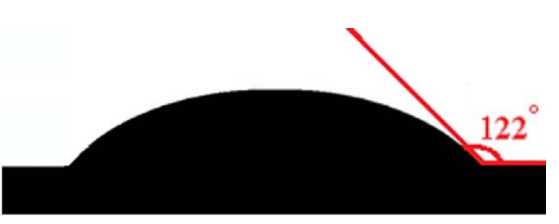

(a2)

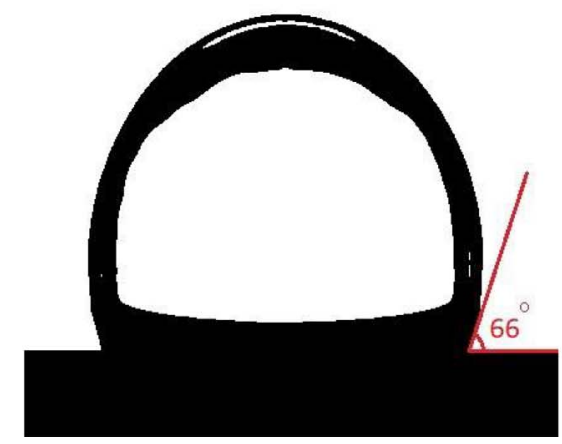

(b2)

Fig. 8. (a) Oil contact angle on the Plexiglass (a1) and 3D printed microfluidic model (a2) showing oil-wet behavior. (b) Water contact angle of the Plexiglass (b1) and 3D printed microfluidic model (b2).

respectively. The result shows that the printed microfluidic model is oil-wet, which makes it superior to the conventional glass micromodels for oil-wet media. Besides, the surface tension result shows that both plexiglass and printed model have very close values. However, the cap sheet of the model can also be $3 \mathrm{D}$ printed with the same material of the body.

\subsection{Numerical modelling setup}

A Computational Fluid Dynamics (CFD) simulator (Ansys Fluent) was employed for the numerical evaluation of the fabricated microfluidic model and to check that the flow paths are not blocked during the printing process. The same structure of the $3 \mathrm{D}$ printed tangible model was used for 
Table 3. Input parameters for the numerical simulations.

\begin{tabular}{lccc}
\hline Parameter & Unit & Miscible simulation & Immiscible simulation \\
\hline Outlet pressure $(P)$ & $\mathrm{Pa}$ & 0 & 0 \\
Flow rate $(Q)$ & $\mathrm{m}^{3} \mathrm{~s}^{-1}$ & $5.6 \mathrm{e}-10$ & $5.6 \mathrm{e}-10$ \\
Dynamic viscosity $(\mu)$ & $\mathrm{Pa} \mathrm{s}^{-3}$ & 0.0010 & 0.0046 \\
Density $(\rho)$ & $\mathrm{kg} \mathrm{m}^{-3}$ & 982 & 755 \\
\hline
\end{tabular}

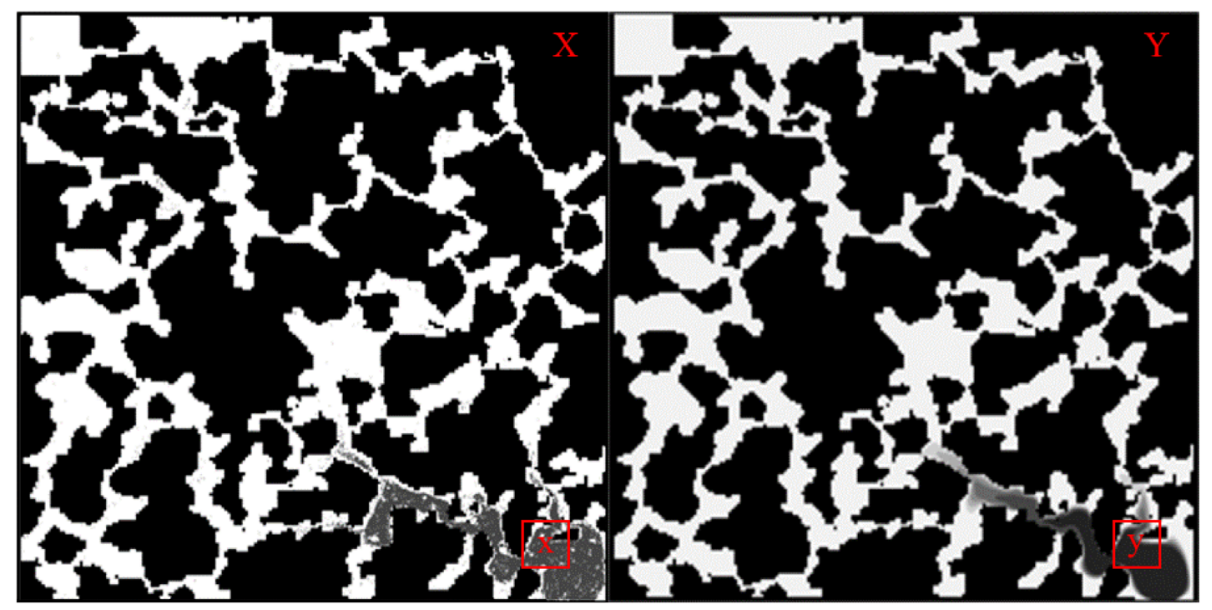

Fig. 9. The 8-bit images of experimental, $X$, (left side) and numerical, $Y$, (right side) result. The red boxes are two sample patches $(x, y)$ of $11 \times 11$ pixels around a center pixel used for comparing the mean, variance, and covariance of both images. These measure patches are computed for all pixels of the image to find the similarity.

CFD simulations. Because of the homogeneity in the $z$-direction, the domain was assumed 2D. The mixture and Volume Of Fluid (VOF) models were used for miscible and immiscible injection processes, respectively. The VOF model can be defined for two or more immiscible liquids. In this model, the fluids share a set of momentum equations for the position of the interface between the fluids. The volume fraction of each fluid is tracked throughout the domain in each computational cell. The mixture and the VOF models differ in three following areas: (i) the inter-phase energy, mass, and momentum transfer are allowed in the mixture model, (ii) the phases can be mixed in the mixture model, and (iii) the mixture model allows the phases to move at different velocities, using the concept of slip velocities $[59,60]$. The difference arises between the average velocities of two different fluids flowing together in a flow path. Hence, the slip velocity depends mainly on the difference in the density between the two fluids, and the diffusivity phenomenon. Practically, the diffusion velocity has to be determined by the slip (relative) velocity which is defined as the velocity of dispersed phase relative to the velocity of the continuous phase [61]. Readers can find further details of the mixture and VOF models in the Ansys Fluent user manual [62].

The following parameters were selected based on the fabricated microfluidic model conditions. The flow rate was considered to be equal to $2 \mathrm{~mL} / \mathrm{h}$, similar to the experiments. The reference velocity can be calculated from the total cross-section of the input port $\left(A=1.56 \times 10^{-6} \mathrm{~m}^{2}\right)$

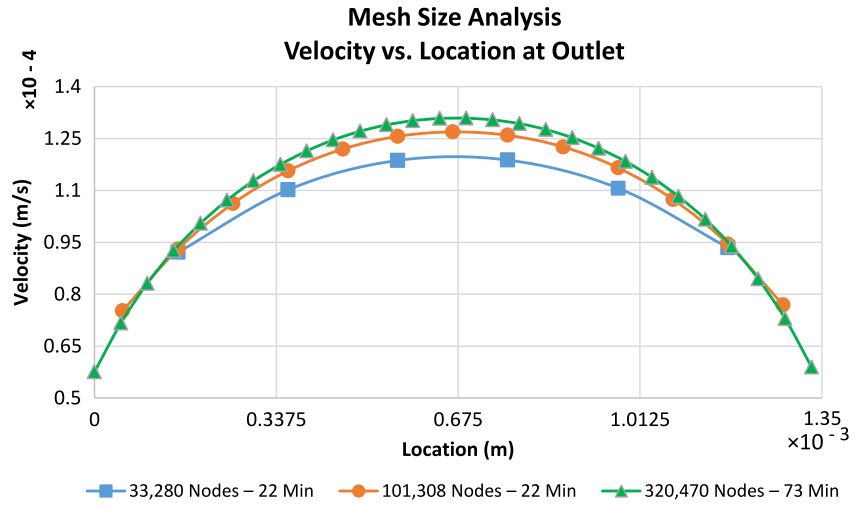

Fig. 10. The velocity profile at the outlet port of the model for mesh independency analysis. The three mesh numbers of 33280 , 101308 , and 320470 have had the simulation run time of 22, 30, and $73 \mathrm{~min}$, respectively.

as $v_{\text {ref }}=Q / A=0.000346 \mathrm{~m} / \mathrm{s}$. Moreover, Reynolds numbers were calculated via $\mathrm{Re}=\rho v d / \mu$ for each miscible and immiscible injection scenario as 0.479 and 0.080 , respectively. Since both are less than 1 , we can be confident that laminar flow regime conditions are satisfied. Since fluid incompressibility was assumed, measuring the absolute pressure value was not necessary; however, the outlet gauge pressure was set to zero. Input and model boundary parameters are summarized in Table 3. 


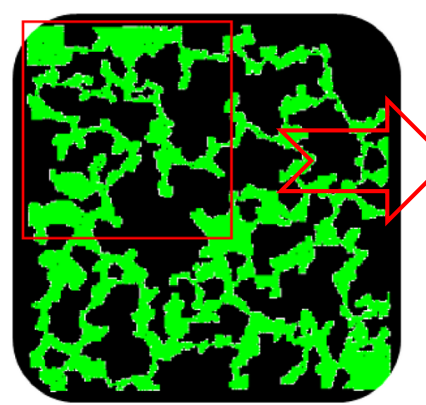

(a)

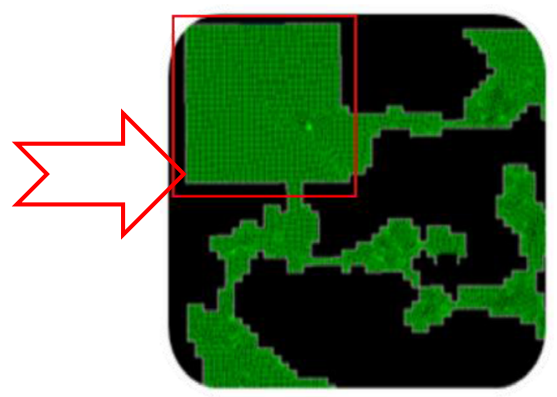

(d)

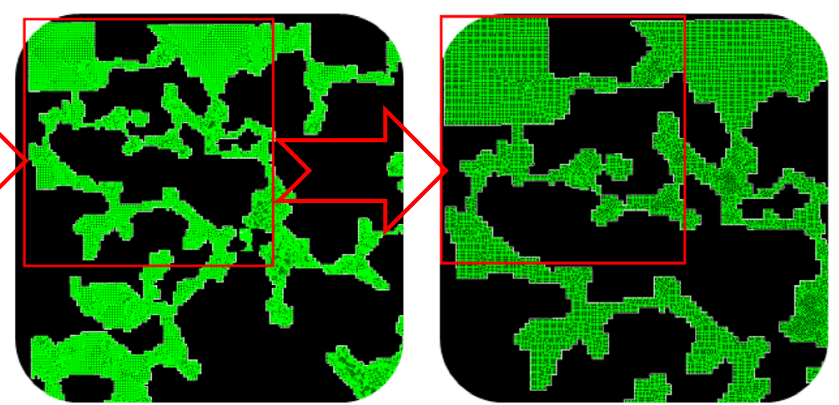

(b)

(c)

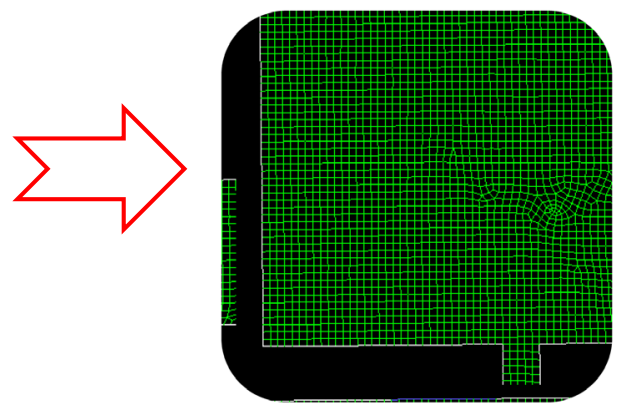

(e)

Fig. 11. Different magnification of the computational domain, (a) $1 \times$ magnitude, (b) $4 \times$ magnitude, (c) $8 \times$ magnitude, (d) $16 \times$ magnitude, (e) $32 \times$ magnitude of (a).

\subsection{Structural Similarity Index Measure (SSIM)}

To validate the accuracy of the microfluidic model, we used a direct comparison of the experimental results with the numerical outcome. The images captured during the injection in the microfluidic model were compared with the numerical simulator predictions at the same time steps of the injection phase following the segmentation of the images through Structural Similarity Index Measure (SSIM) [63]. It is worth mentioning that all images have been converted to 8-bit black and white images (Fig. 9). This index has been used in related studies widely [64, 65].

The comparison between the two $X$ and $Y$ images is performed on the basis of these 3 features; luminance $(l)$, contrast $(c)$, and structure $(s)$.

The SSIM compares the luminance, contrast, and structure of two $X$ and $Y$ images locally to quantify the similarity between them. The SSIM index is a decimal value between 0 and 1 where 1 shows a perfect structural similarity, and it happens only when two completely identical images are being compared. A patch of $11 \times 11$ pixels around a center pixel is selected for computing the properties of each center pixel (red boxes in Fig. 9). Hence, the SSIM between two $x$ and $y$ patches of both $X$ and $Y$ images are defined as [66],

$$
\operatorname{SSIM}=l(x . y) \times c(x . y) \times s(x . y),
$$

where,

$$
l(x . y)=\frac{2 \mu_{x} \mu_{y}+C_{1}}{\mu_{x}^{2}+\mu_{y}^{2}+C_{1}}
$$

$$
\begin{gathered}
c(x . y)=\frac{2 \sigma_{x} \sigma_{y}+C_{2}}{\sigma_{x}^{2}+\sigma_{y}^{2}+C_{2}}, \\
s(x . y)=\frac{\sigma_{x y}+C_{3}}{\sigma_{x} \sigma_{y}+C_{3}},
\end{gathered}
$$

therefore;

$$
\operatorname{SSIM}(x . y)=\frac{\left(2 \mu_{x} \mu_{y}+C_{1}\right)\left(2 \sigma_{x y}+C_{2}\right)}{\left(\mu_{x}^{2}+\mu_{y}^{2}+C_{1}\right)\left(\sigma_{x}^{2}+\sigma_{y}^{2}+C_{2}\right)},
$$

where $\mu_{x}$ and $\mu_{y}$ are the average value of $x$ and $y$ patches, respectively, $\sigma_{x}^{2}$ and $\sigma_{y}^{2}$ are the variance of each $x$ and $y$, and $\sigma_{x y}$ is the covariance of the $x$ and $y$ patches:

$$
\begin{gathered}
\mu_{x}=\frac{1}{N} \sum_{i=1}^{N} x_{i}, \quad \text { and } \quad \mu_{y}=\frac{1}{N} \sum_{i=1}^{N} y_{i}, \\
\sigma_{x}=\left(\frac{1}{N-1} \sum_{i=1}^{N}\left(x_{i}-\mu_{x}\right)^{2}\right)^{\frac{1}{2}}, \quad \text { and } \\
\sigma_{y}=\left(\frac{1}{N-1} \sum_{i=1}^{N}\left(y_{i}-\mu_{y}\right)^{2}\right)^{\frac{1}{2}},
\end{gathered}
$$

$$
\sigma_{x y}=\frac{1}{N-1}\left(\sum_{i=1}^{N}\left(\left[x_{i}-\mu_{x}\right] \times\left[y_{i}-\mu_{y}\right]\right)\right) .
$$




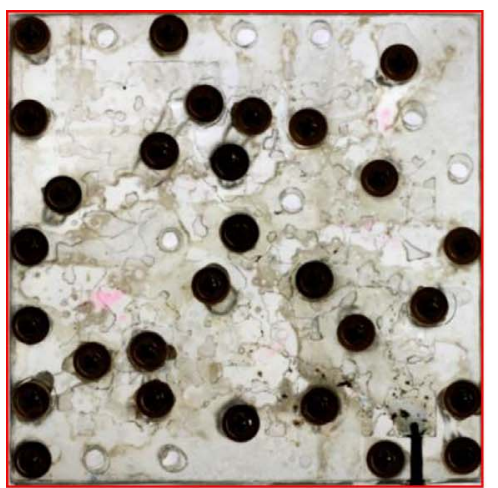

A

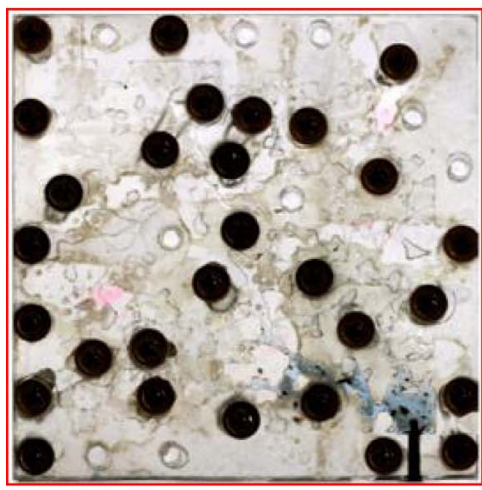

B

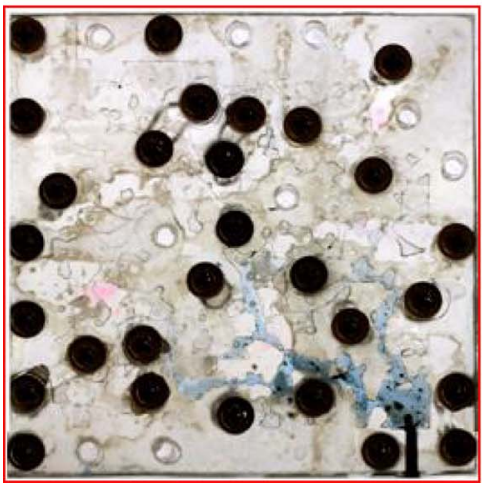

C

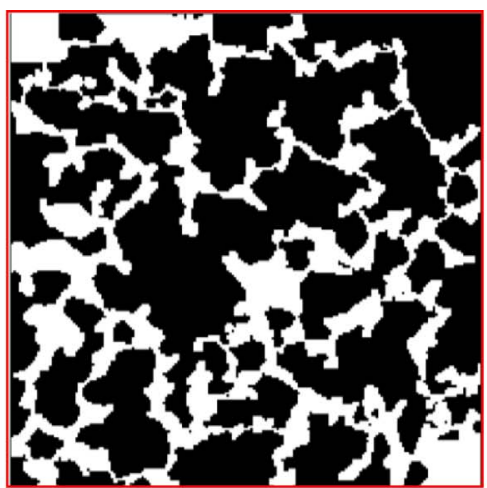

F

(a)

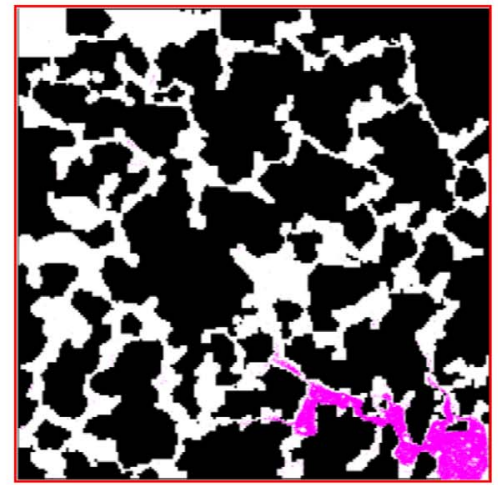

G

(b)

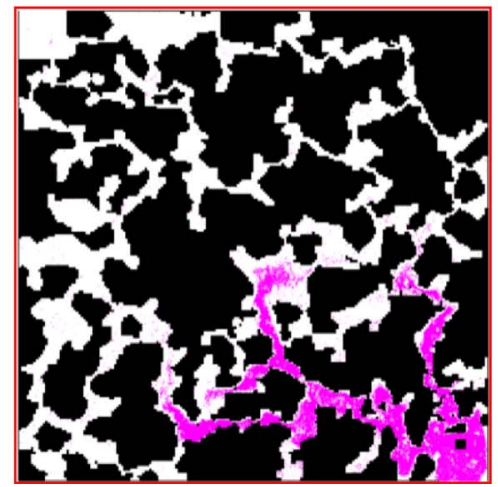

$\mathrm{H}$

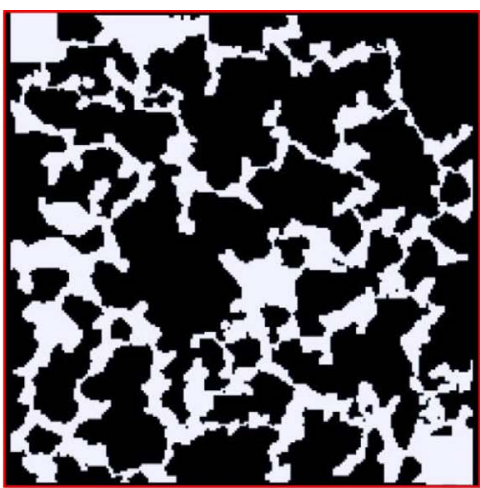

K

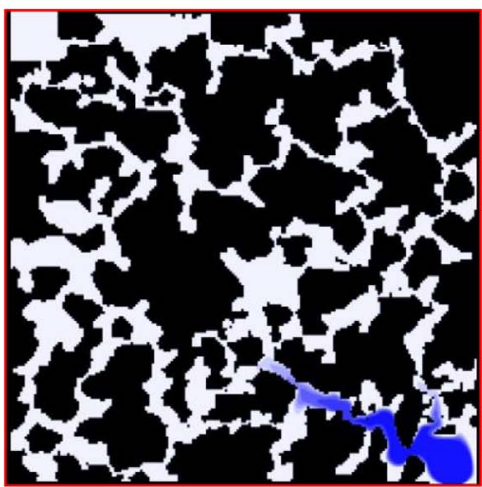

L

(c)

Fig. 12. The captured images of the miscible flow scenario at different time intervals. The left, middle, and right columns show the experiment images, the segmented images of experiments, and numerical simulation results, respectively. (a) Initial condition, (b) $140 \mathrm{~s}$, (c) $340 \mathrm{~s}$, (d) $540 \mathrm{~s}$, and (e) $1300 \mathrm{~s}$ (i.e., breakthrough time). The colored areas (pink and blue) represent the injected fluid; the white areas are pores, and the black areas representing solid grains.

$C_{1}, C_{2}$ and $C_{3}$ are three constants to ensure stability when the denominator of all fractions becomes 0 and given by,

$$
\begin{aligned}
& C_{1}=\left(K_{1} L\right)^{2}, \\
& C_{2}=\left(K_{2} L\right)^{2},
\end{aligned}
$$

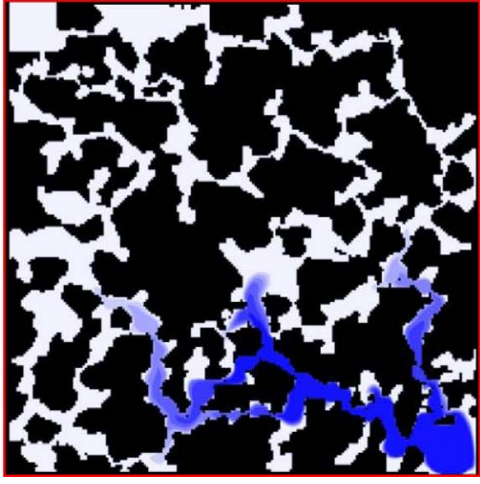

M 


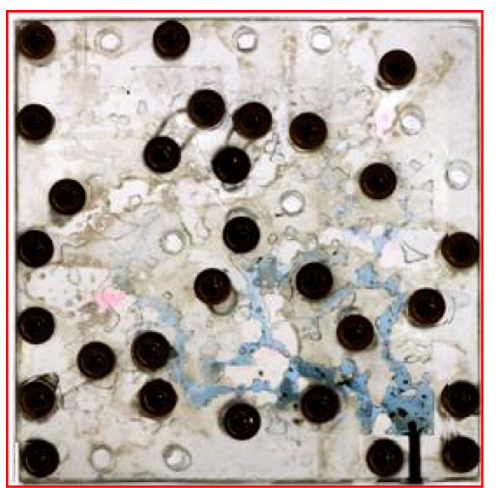

D

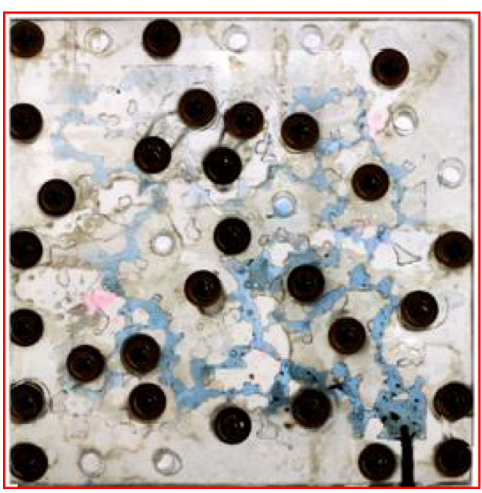

$\mathrm{E}$

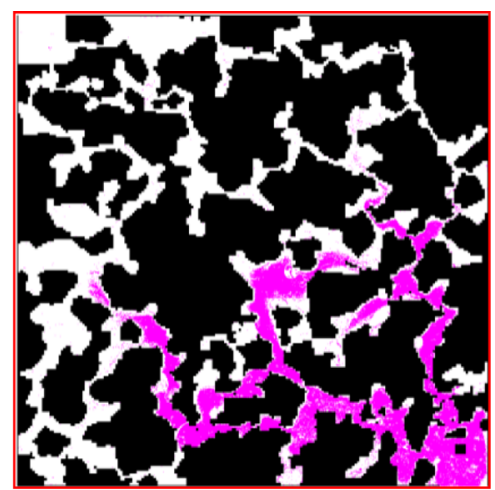

(

(d)

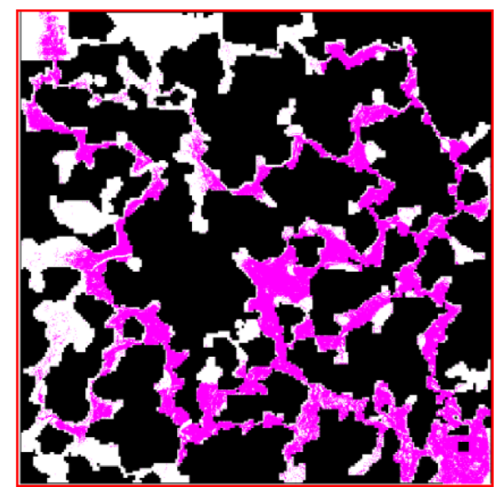

$\mathrm{J}$

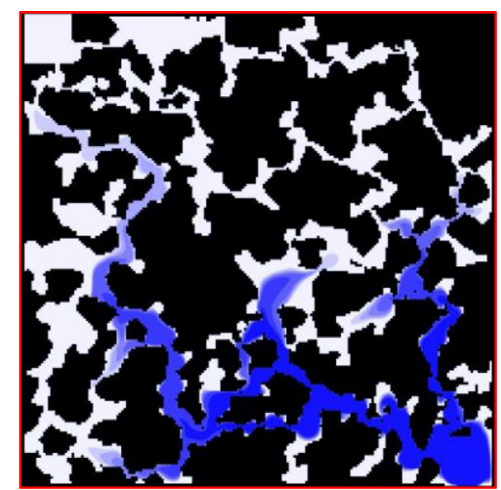

$\mathrm{N}$

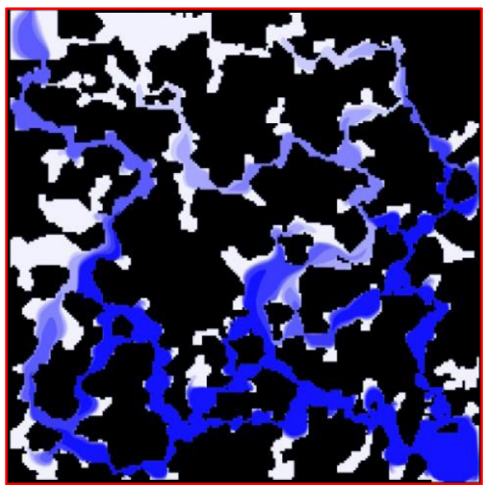

O

(e)

Fig. 12. Continued.

\section{Results}

\subsection{Mesh-size}

The pore space was populated with a different number of cells (i.e., 33 280, 101308 , and 320 470) to numerically investigate the mesh dependency of the model. Figure 10 illustrates the velocity profile at the outlet port of the microfluidic model with a different number of cells and associated simulation run time on an Inte ${ }^{\circledR}$ Core $^{T M}$ i7, 6700HQ processor. Uncertainty analysis is a systematic study performed to assess the consistency and accuracy of a CFD solver in solving the intended problem. Uncertainty analysis is sub-divided into two processes, verification and validation. Verification assesses the consistency of the solver, whereas, validation evaluates its accuracy. It is expected that the accuracy of results and the simulation run time are increased by raising the number of mesh cells. However, above a mesh number the simulation results remain constant and no changes are observable. The velocity profile prediction of the simulation model with 320470 mesh does not differ much from the model comprised of 101308 cells (the velocity variation is less than 4\%). Thus, the grid mesh with 101308 mesh cells was used for further
Table 4. The SSIM index of miscible scenarios showing good agreement between the experimental and numerical predictions.

\begin{tabular}{lcc}
\hline Time $(\mathrm{s})$ & SSIM & Normalized SSIM \\
\hline 0 & 0.95 & 1 \\
140 & 0.94 & 0.98 \\
340 & 0.91 & 0.96 \\
540 & 0.89 & 0.94 \\
1300 & 0.86 & 0.90 \\
\hline
\end{tabular}

simulations. Figure 11 depicts the final mesh structure of the microfluidic model at different mesh sizes and locations within the microfluidic model.

\subsection{Miscible injection scenario}

Several images were captured during the entire injection process. Figures $12 \mathrm{~A}-12 \mathrm{E}$ depict the distribution of fluids obtained at different time intervals $(0,140,240,540$, and $1300 \mathrm{~s}$ ) during the miscible injection scenario. The displaced 


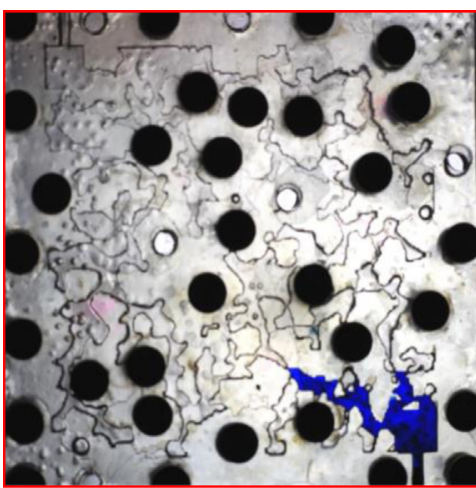

A

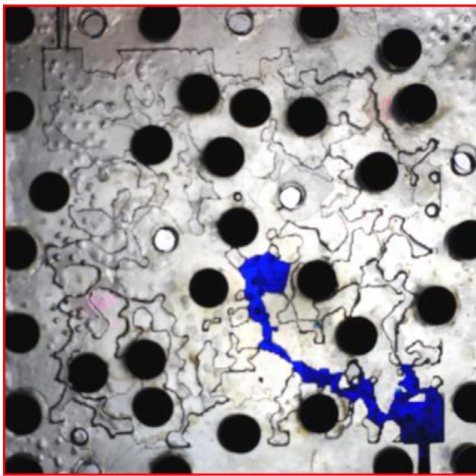

B

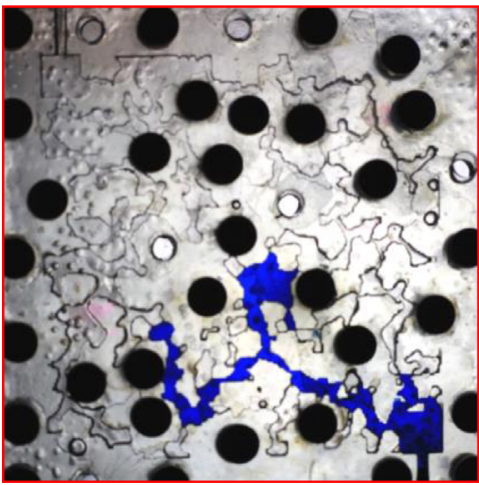

C

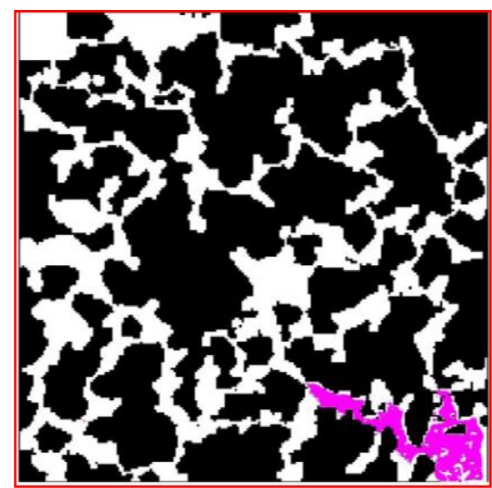

$\mathrm{F}$

(a)

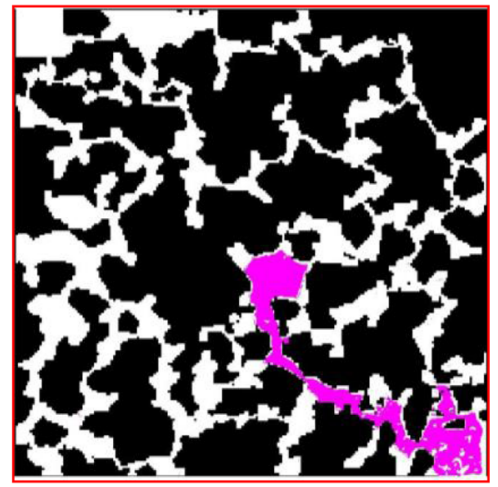

G

(b)

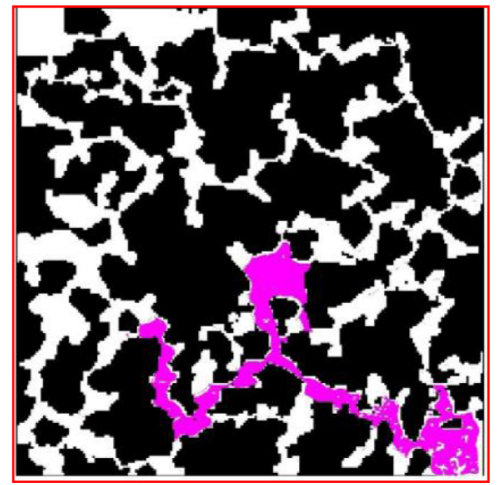

$\mathrm{H}$

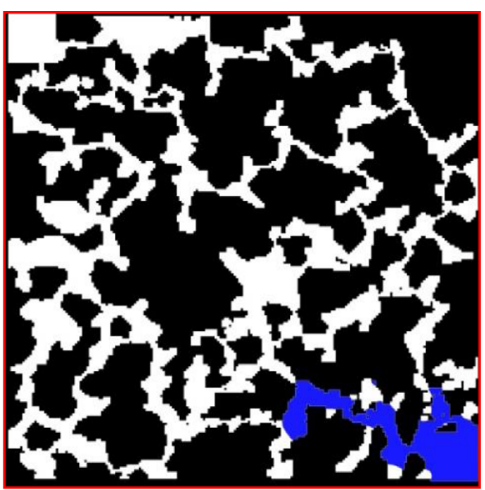

$\mathrm{K}$

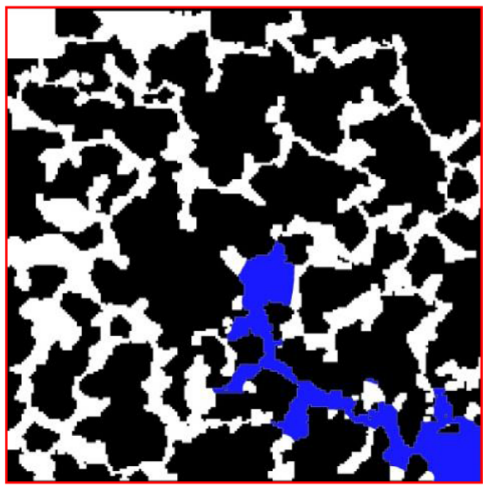

$\mathrm{L}$

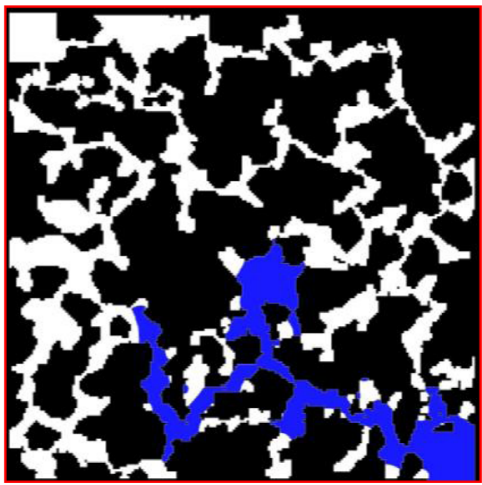

$\mathrm{M}$

(c)

Fig. 13. The captured images of the immiscible flow scenario at different time intervals. The left, middle, and right columns show the experiment images, the segmented images of the experiment, and numerical simulation results, respectively. (a) $35 \mathrm{~s}$, (b) $70 \mathrm{~s}$, (c) $100 \mathrm{~s}$, (d) $120 \mathrm{~s}$, and (e) $133 \mathrm{~s}$ (i.e., breakthrough time). The colored areas (pink and blue) represent the injected fluid; the white areas are pores, and the black areas representing solid grains.

and displacing fluids are shown colorless and in blue, respectively. Figures $12 \mathrm{~F}-12 \mathrm{~J}$ show the binary images of the experimental captured photos analyzed by the ImageJ. It should be mentioned that pink pixels are representing the injected blue-dyed water. Moreover, the prediction of fluid distributions obtained from the numerical simulation of the same microfluidic model is depicted in Figures $12 \mathrm{~K}$ 12O. As discussed in the previous section, the mixture model was used to simulate this tracer test process (two miscible fluids). Figure 12a shows the microfluidic model at the beginning of an injection test $(t=0 \mathrm{~s})$ when it is fully saturated with deionized water. Since the injection process 


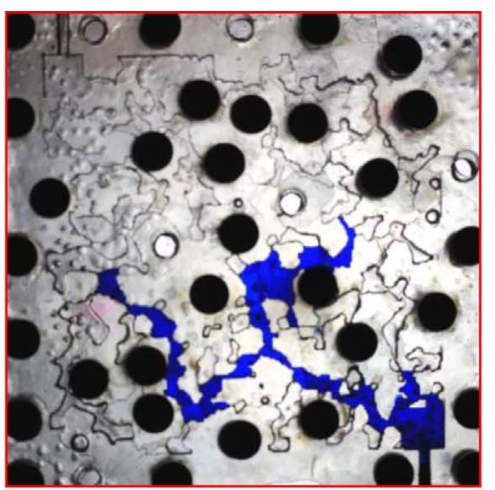

D

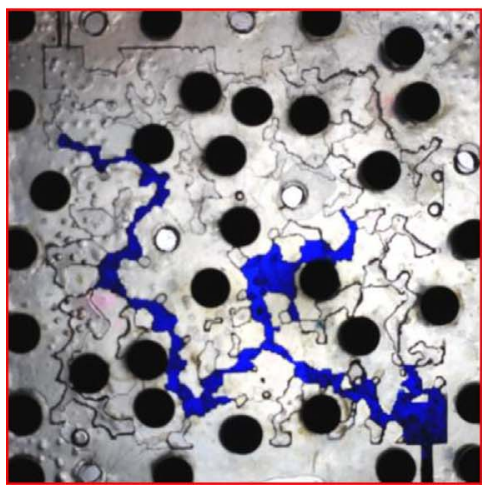

$\mathrm{E}$

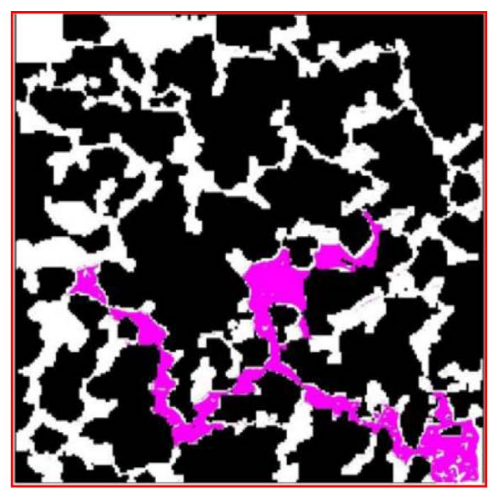

I

(d)

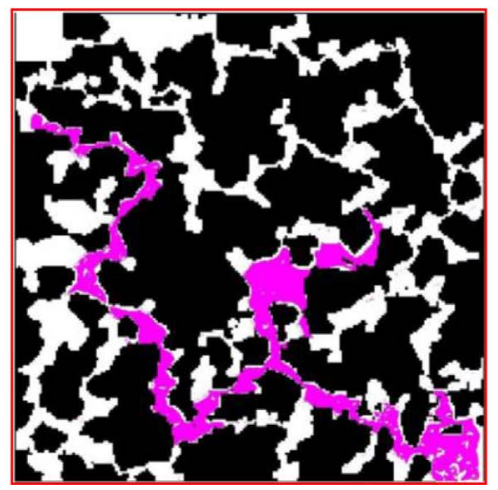

$\mathrm{J}$

(e)

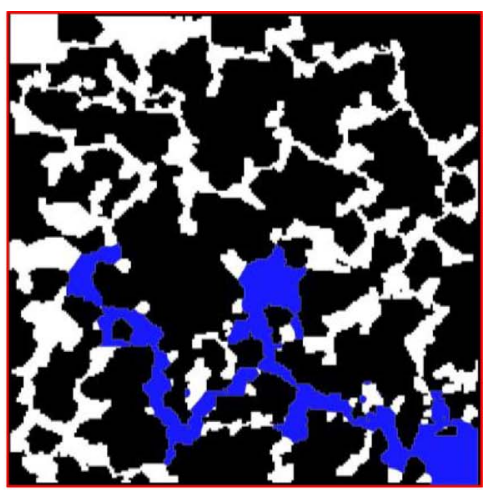

$\mathrm{N}$

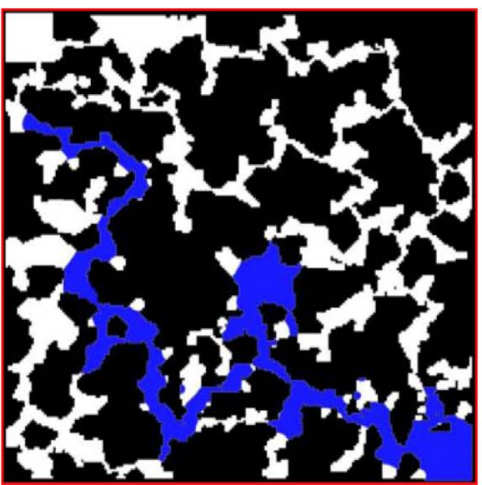

O

Fig. 13. Continued.

is miscible, when the colored tracer fluid is injected, both phases would mix. As a result, the injected fluid flew relatively uniform throughout the entire porous medium, and the breakthrough happened at $1300 \mathrm{~s}$ after the start of injection.

Table 4 summarizes the SSIM calculated for the experiments and simulations. The normalized SSIM value of the initial time step is defined as:

$$
\text { Normalized } \mathrm{SSIM}=\frac{\operatorname{SSIM}_{I}}{\operatorname{SSIM}_{\mathrm{at} t=0}} .
$$

Based on Table 4, high similarity values between experimental and numerical results can be observed. Therefore, a good agreement between the results during the miscible injection scenario is obtained. This confirms that the process of fabricating the flow pathways through the introduced 3D printing approach was successful because flow patterns on digital and tangible analogs were similar at the pore scale (Fig. 12).

\subsection{Immiscible injection scenario}

The entire process of the immiscible injection scenario is repeated just the same as the miscible scenario except before starting the experiment; the microfluidic model was saturated with synthetic oil. To simulate the immiscible injection scenario, the VOF model was used under laminar conditions. The images that are captured during the experiments, the corresponding segmented images, and the numerical predictions are shown in $\mathrm{A}-\mathrm{E}, \mathrm{F}-\mathrm{J}$, and $\mathrm{K}-\mathrm{O}$ in Figure 13, respectively. The captured images at five different time steps $(35,70,100,120$, and $133 \mathrm{~s})$ are shown for comparison purposes in this figure.

Comparing the binary images from the experiments with numerical simulation results in Figure 13, it was found that a visual similarity of flow streams in both cases is attained. The SSIM comparing the experiments and simulations is summarized in Table 5 . The structural similarity of both experimental and numerical results at the same time intervals was calculated to be above $88 \%$, which reflects a high resemblance between the experimental and numerical results (normalized SSIM > 0.95) during the immiscible injection scenario. This similarity again represents that implementing our printed technology to fabricate the microfluidic model was reliable, and the printed model at the pore-scale has similar behavior to the rock analog that was based on the CT data of a natural specimen.

Figure 13 explains that during the immiscible flooding scenario, oil production took place mostly before the breakthrough time (133 s). After the breakthrough happens, the 
Table 5. The SSIM index between the immiscible scenario images of the experiments and simulations at different time intervals.

\begin{tabular}{lcc}
\hline Time $(\mathrm{s})$ & SSIM & Normalized SSIM \\
\hline 35 & 0.92 & 0.99 \\
70 & 0.92 & 0.98 \\
100 & 0.91 & 0.97 \\
120 & 0.89 & 0.95 \\
133 & 0.89 & 0.95 \\
\hline
\end{tabular}

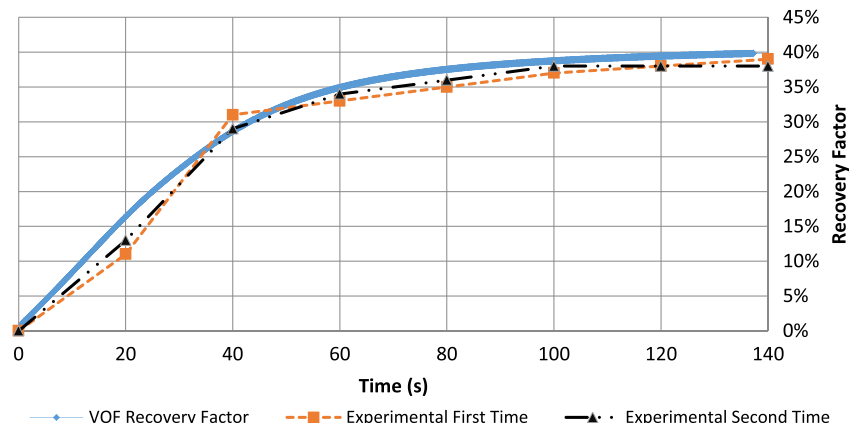

Fig. 14. The oil recovery factor of the immiscible injection scenario.

displacing fluid only will flow through the same path within the pore space and exit the microfluidic model without any further oil displacement on new routes. The recovery factor at various time steps of the experiments was calculated by measuring the number of colored pixels of the displacing fluid divided by the total area of the microfluidic model that is filled with the displaced fluid. Results exhibit that the recovery factor increased until the breakthrough time is achieved, and then stayed stagnant. To ensure the accuracy of the experiments, the process was repeated twice (setup was cleaned entirely before the second round), and results are compared with the predictions of the VOF model, shown in Figure 14. The final recovery factor was $40 \pm 3 \%$, while an excellent agreement between VOF with both rounds of the experiments is achieved.

As the results show, unlike the previous studies $[9,16$, 49], a more complex geometry - adopted from rock CT scanned images - has been used in this study. The fabrication materials was oil-wet initially as opposed to the conventional glass micromodels that need additional steps for wettability alteration of models [10, 11]. Furthermore, if the setup is used in destructive experiments [23, 24], repeatability of experiments on reproducible microfluidic devices is the main motive of using such $3 \mathrm{D}$ printed models.

\section{Conclusion}

This study investigated the pore-scale flow through a heterogeneous carbonate rock using the digital-light processing $3 \mathrm{D}$ printing technology through direct observation of the results. The idea was to manufacture a reproducible transparent microfluidic model directly from actual rock. A comprehensive guideline was presented to verify the quality of the printed microfluidic model using parallel CFD simulation of true flooding processes on the printed model images. This microfluidic model was subjected to single and multiphase injection scenarios. Furthermore, the same processes were simulated by a CFD numerical simulator to ensure the flow paths are not blocked during the printing process. Flow stream comparison of the segmented experimental images with the numerical simulation predictions in both miscible and immiscible scenarios indicated high similarities in our relatively complex pore geometry. The obtained results from the CFD simulations and the experimental data were compared in $2 \mathrm{D}$ by a similarity measure index, known as SSIM. The normalized measured index showed an acceptable similarity of both miscible $(>90 \%)$ and immiscible $(>95 \%)$ injection scenarios. Thus, it can be concluded that fabricating the microfluidic model by the DLP 3D printing technology from the CT-images of the natural rock samples looks promising, and this technology can be an appropriate tool for reproducing microfluidic porous media in the future. Reproducing reusable porous media with known properties has a variety of applications in the petroleum industry, especially in analyzing reactive flow in porous media. The authors acknowledge that the current printing technology still has limitations in terms of resolution, overall device dimensions, and resin versatility for porescale flow analysis. However, the introduced guideline for printing 2.5D micromodels can provide potential for fluid flow studies.

\section{References}

1 Blunt M.J. (2017) Multiphase flow in permeable media: A porescale perspective, Cambridge University Press, Cambridge, UK.

2 Rostami P., Sharifi M., Aminshahidy B., Fahimpour J. (2019) The effect of nanoparticles on wettability alteration for enhanced oil recovery: Micromodel experimental studies and CFD simulation, Petrol. Sci. 16, 4, 859-873.

3 Palizdan S., Doryani H., Riazi M., Malayeri M.R. (2020) Experimental study of in-situ $\mathrm{W} / \mathrm{O}$ emulsification during the injection of $\mathrm{MgSO}_{4}$ and $\mathrm{Na}_{2} \mathrm{CO}_{3}$ solutions in a glass micromodel, Oil Gas Sci. Technol. - Rev. IFP Energies nouvelles $\mathbf{7 5}, 87$.

4 Mahmoodi M., Mahdavi S., James L.A., Johansen T. (2018) A quick method to fabricate large glass micromodel networks, Microsyst. Technol. 24, 5, 2419-2427.

5 Chuoke R., Van Meurs P., van der Poel C. (1959) The instability of slow, immiscible, viscous liquid-liquid displacements in permeable media, Trans. AIME 216, 01, 188-194.

6 Yi S., Babadagli T., Li H. (2020) Stabilization of nickel nanoparticle suspensions with the aid of polymer and surfactant: Static bottle tests and dynamic micromodel flow tests, Petrol. Sci. 17, 1-11.

7 Muraoka M., Yamamoto Y., Tenma N. (2020) Simultaneous measurement of water permeability and methane hydrate pore habit using a two-dimensional glass micromodel, J. Nat. Gas Sci. Eng. 77, 103279. 
8 Abolhasanzadeh A., Khaz'ali A.R., Hashemi R., Jazini M. (2020) Experimental study of microbial enhanced oil recovery in oil-wet fractured porous media, Oil Gas Sci. Technol. - Rev. IFP Energies nouvelles 75, 73.

9 Meisenheimer D.E., McClure J.E., Rivers M.L., Wildenschild D. (2020) Exploring the effect of flow condition on the constitutive relationships for two-phase flow, $A d v$. Water Res. 137, 103506.

10 Cao S.C., Jung J., Radonjic M. (2019) Application of microfluidic pore models for flow, transport, and reaction in geological porous media: From a single test bed to multifunction real-time analysis tool, Microsyst. Technol. 25, 1-18.

11 Lin M., Wang Y., Cao Y., Wang Y., Wang X., Xi K. (2020) Experimental study of the influence of oil-wet calcite cements on oil migration and implications for clastic reservoirs, Mar. Pet. Geol. 118, 104427.

12 Song Y., Zhao C., Chen M., Chi Y., Zhang Y., Zhao J. (2020) Pore-scale visualization study on $\mathrm{CO}_{2}$ displacement of brine in micromodels with circular and square cross sections, Int. J. Greenhouse Gas Cont. 95, 102958.

13 Sharifipour M., Nakhaee A., Pourafshary P. (2019) Model development of permeability impairment due to clay swelling in porous media using micromodels, J. Pet. Sci. Eng. $\mathbf{1 7 5}$ 728-742.

14 Porter M.L., Jiménez-Martínez J., Martinez R., McCulloch Q., Carey J.W., Viswanathan H.S. (2015) Geo-material microfluidics at reservoir conditions for subsurface energy resource applications, Lab. Chip 15, 20, 4044-4053.

15 Martel R., Portois C., Robert T., Uyeda M. (2019) Etched glass micromodel for laboratory simulation of NAPL recovery mechanisms by surfactant solutions in fractured rock, J. Contam. Hydrol. 227, 103550.

16 Watson F., Maes J., Geiger S., Mackay E., Singleton M., McGravie T., Anouilh T., Jobe T.D., Zhang S., Agar S., Ishutov S. (2019) Comparison of flow and transport experiments on 3D printed micromodels with direct numerical simulations, Transp. Porous Media 129, 2, 449-466.

17 Aziz R., Niasar V., Erfani H., Martínez-Ferrer P.J. (2020) Impact of pore morphology on two-phase flow dynamics under wettability alteration, Fuel 268, 117315.

18 Song W., de Haas T.W., Fadaei H., Sinton D. (2014) Chipoff-the-old-rock: The study of reservoir-relevant geological processes with real-rock micromodels, Lab. Chip 14, 22, 4382-4390.

19 Tanino Y., Zacarias-Hernandez X., Christensen M. (2018) Oil/water displacement in microfluidic packed beds under weakly water-wetting conditions: Competition between precursor film flow and piston-like displacement, Exp. Fluids $\mathbf{5 9}, 2,35$.

20 Zhang Y., Sanati-Nezhad A., Hejazi S. (2018) Geo-material surface modification of microchips using Layer-By-Layer (LbL) assembly for subsurface energy and environmental applications, Lab. Chip 18, 2, 285-295.

21 Singh R., Sivaguru M., Fried G.A., Fouke B.W., Sanford R.A., Carrera M., Werth C.J. (2017) Real rock-microfluidic flow cell: A test bed for real-time in situ analysis of flow, transport, and reaction in a subsurface reactive transport environment, J. Contam. Hydrol. 204, 28-39.

22 Teimouri A., Sadeghnejad S., Dehaghani A.H.S. (2020) Investigation of acid pre-flushing and $\mathrm{pH}$-sensitive microgel injection in fractured carbonate rocks for conformance control purposes, Oil Gas Sci. Technol. - Rev. IFP Energies nouvelles $\mathbf{7 5}, 52$.
23 Pratama R.A., Babadagli T. (2020) Wettability state and phase distributions during steam injection with and without chemical additives: An experimental analysis using visual micromodels, SPE Reserv. Eval. Eng. 23, 1133-1149.

24 Tahir M., Hincapie R.E., Gaol C.L., Säfken S., Ganzer L. (2020) Flow dynamics of sulfate-modified water/polymer flooding in micromodels with modified wettability, Appl. Sci. 10, 9,3239 .

25 Song W., Kovscek A.R. (2016) Direct visualization of porescale fines migration and formation damage during lowsalinity waterflooding, J. Nat. Gas Sci. Eng. 34, 1276-1283.

26 Zhang Y., Yesiloz G., Sharahi H.J., Khorshidian H., Kim S., Sanati-Nezhad A., Hejazi S.H. (2019) Geomaterialfunctionalized microfluidic devices using a universal surface modification approach, Adv. Mater. Interf. 6, 23, 1900995.

27 Hull C.W. (1984) Apparatus for production of threedimensional objects by stereolithography. United States Patent, Appl., No. 638905, Filed.

28 Sing S.L., Tey C.F., Tan J.H.K., Huang S., Yeong W.Y. (2020) 3D printing of metals in rapid prototyping of biomaterials: Techniques in additive manufacturing, in: Rapid prototyping of biomaterials, Elsevier, Amsterdam, pp. $17-40$.

29 Yadav D.K., Srivastava R., Dev S. (2020) Design \& fabrication of ABS part by FDM for automobile application, Mater. Today Proc. 26, 2089-2093.

30 Shahrubudin N., Lee T., Ramlan R. (2019) An overview on $3 \mathrm{D}$ printing technology: Technological, materials, and applications, Procedia Manuf. 35, 1286-1296.

31 Craveiro F., Nazarian S., Bartolo H., Bartolo P.J., Duarte J.P. (2020) An automated system for 3D printing functionally graded concrete-based materials, Addit. Manuf. 33, 101146.

32 Dmitriev A.Y., Zagidulin R., Mitroshkina T. (2020) Special aspects of quality assurance in the design, manufacture, testing of aerospace engineering products, in: IOP Conference Series: Materials Science and Engineering, IOP Publishing, Bristol, UK.

33 Dang W., Ma B., Li B., Huan Z., Ma N., Zhu H., Chang J., Xiao Y., Wu C. (2020) 3D printing of metal-organic framework nanosheets-structured scaffolds with tumor therapy and bone construction, Biofabrication 12, 2, 025005.

34 Ishutov S., Jobe T.D., Zhang S., Gonzalez M., Agar S.M., Hasiuk F.J., Watson F., Geiger S., Mackay E., Chalaturnyk R. (2018) Three-dimensional printing for geoscience: Fundamental research, education, and applications for the petroleum industry, AAPG Bull. 102, 1, 1-26.

35 Wu T., Zhao H., Xu Q., Zhao Y. (2020) Optimal analysis of material ratio for artificial rock by $3 \mathrm{D}$ printing technique, Geomech. Geoeng. 15, 1-9.

$36 \mathrm{Li} \mathrm{H.}$, Zhang T. (2019) Imaging and characterizing fluid invasion in micro-3D printed porous devices with variable surface wettability, Soft Matter 15, 35, 6978-6987.

37 Shallan A.I., Smejkal P., Corban M., Guijt R.M., Breadmore M.C. (2014) Cost-effective three-dimensional printing of visibly transparent microchips within minutes, Anal. Chem. 86, 6, 3124-3130.

38 Ishutov S. (2017) 3D printing porous proxies as a new tool for laboratory and numerical analyses of sedimentary rocks, PhD Dissertation, Iowa State University, Geological and Atmospheric Sciences Department.

39 Perras M.A., Vogler D. (2019) Compressive and tensile behavior of 3D-Printed and natural sandstones, Trans. Porous Media 129, 2, 559-581. 
40 Hodder K.J., Nychka J.A. (2019) Silane treatment of 3 D-printed sandstone models for improved spontaneous imbibition of water, Trans. Porous Media 129, 2, 583-598.

41 Kong L., Ostadhassan M., Li C., Tamimi N. (2018) Pore characterization of 3D-printed gypsum rocks: a comprehensive approach, J. Mater. Sci. 53, 7, 5063-5078.

42 Kong L., Ostadhassan M., Li C., Tamimi N. (2017) Rock physics and geomechanics of 3-D printed rocks, in: 51st US Rock Mechanics/Geomechanics Symposium, American Rock Mechanics Association.

43 Kong L., Ostadhassan M., Li C., Tamimi N. (2018) Can 3-D printed gypsum samples replicate natural rocks? An experimental study, Rock Mech. Rock Eng. 51, 10, 30613074 .

44 Kong L., Ostadhassan M., Hou X., Mann M., Li C. (2019) Microstructure characteristics and fractal analysis of 3Dprinted sandstone using micro-CT and SEM-EDS, J. Pet. Sci. Eng. 175, 1039-1048.

45 Almetwally A., Jabbari H. (2019) Development of novel workflow to replicate pore network of porous core samples through 3D printing technology, in: 53rd US Rock Mechanics/ Geomechanics Symposium, American Rock Mechanics Association.

46 Almetwally A., Jabbari H. (2020) Experimental investigation of 3D printed rock samples replicas, J. Nat. Gas Sci. Eng. 76, 103192.

47 Song R., Peng J., Sun S., Wang Y., Cui M., Liu J. (2020) Visualized experiments on residual oil classification and its influencing factors in waterflooding using micro-computed tomography, J. Energy Resour. Technol. 142, 8, 083003.

48 Hasiuk F.J. (2019) Testing bulk properties of powder-based 3D-printed reservoir rock proxies, Trans. Porous Media 129, 2, 501-520.

49 Yang W., Zhang D., Lei G. (2020) Experimental study on multiphase flow in fracture-vug medium using 3D printing technology and visualization techniques, J. Pet. Sci. Eng. 193, 107394

50 Donovan K.J. (2019) Microfluidic investigations of capillary flow and surface phenomena in porous polymeric media for 3D printing, PhD Dissertation, Oregon State University, Materials Science Department.

51 Ahkami M., Roesgen T., Saar M.O., Kong X.Z. (2019) Highresolution temporo-ensemble PIV to resolve pore-scale flow in 3D-printed fractured porous media, Trans. Porous Media 129, 2, 467-483.

52 Moslemipoor A. (2019) Pore Network Modeling (PNM) of vuggy carbonate reservoirs and its validation by experimental data, in: Department of Chemical Engineering, Tarbiat Modares University.

53 Moslemipour A., Sadeghnejad S. (2020) Dual-scale pore network reconstruction of vugular carbonates using multiscale imaging techniques, Adv. Water Resour. 147, 103795.

54 Schindelin J., Arganda-Carreras I., Frise E., Kaynig V., Longair M., Pietzsch T., Preibisch S., Rueden C., Saalfeld S., Schmid B., Tinevez J.Y. (2012) Fiji: An open-source platform for biological-image analysis, Nat. Meth. 9, 7, 676-682.

55 Esmaeilzadeh S., Salehi A., Hetz G., Olalotiti-lawal F., Darabi H., Castineira D. (2020) Multiscale modeling of compartmentalized reservoirs using a hybrid clusteringbased non-local approach, J. Pet. Sci. Eng. 184, 106485.

56 Liew A.W.L. (2020) Laser-based 3D printing using bessel beams for tissue engineering applications, PhD Dissertation, Nanyang Technological University, School of Mechanical and Aerospace Engineering.

57 Kim B., Kim H., Kim J., Cho C.S., Lee J. (2013) Superhydrophobic polytetrafluoroethylene surface obtained using reactive ion etching and duplication with polydimethylsiloxane mould, Micro Nano Lett. 8, 10, 691-695.

58 Steingruber E. (2000) Indigo and indigo colorants, in: Ullmann's Encyclopedia of Industrial Chemistry, Wiley$\mathrm{VCH}$, Weinheim, Germany.

59 Eghbalzadeh A., Javan M. (2012) Comparison of mixture and VOF models for numerical simulation of air-entrainment in skimming flow over stepped spillways, Procedia Eng. 28, 657-660.

60 Hirt C.W., Nichols B.D. (1981) Volume Of Fluid (VOF) method for the dynamics of free boundaries, J. Comput. Phys. 39, 1, 201-225.

61 Manninen M., Taivassalo V. (1996) On the mixture model for multiphase flow, VTT Publications 288, Technical Research Center of Finland, Finland.

62 Fluent A. (2009) 12.0 Theory guide, Ansys Inc. 5, 5, 15.

63 Wang Z., Bovik A.C. (2002) A universal image quality index, IEEE Sig. Proc. Lett. 9, 3, 81-84.

64 Okarma K., Fastowicz J., Tecõõlaw M. (2016) Application of structural similarity based metrics for quality assessment of 3D prints, in: International Conference on Computer Vision and Graphics, Springer.

65 Yuan J., Tian J., Chen C., Chen G. (2020) Experimental investigation of color reproduction quality of color $3 \mathrm{D}$ printing based on colored layer features, Molecules 25, 12, 2909.

66 Wang Z., Bovik A.C., Sheikh H.R., Simoncelli E.P. (2004) Image quality assessment: From error visibility to structural similarity, IEEE Trans. Image Proc. 13, 4, 600-612. 